\title{
EDEMA PULMONAR EN CIRUGÍA LAPAROSCÓPICA. PRESENTACIÓN DE UN CASO Y REVISIÓN SISTEMÁTICA DE LA LITERATURA
}

\author{
Pulmonary edema in laparoscopic surgery. \\ Case report and systematic review \\ of the literature \\ Javier Eslava, M.D., M.Sc*, Hernando Gaitán, M.D., M.Sc**, Nelson Pedraza, M.D.**** \\ Recibido: septiembre 21/2005 - Revisado: octubre 13/2005 - Aceptado: diciembre 27/2005
}

\section{RESUIMEN}

La cirugía laparoscópica es una técnica que se ha difundido exponencialmente y a la que se le han reconocido muchas bondades. Sin embargo, también se le ha asociado una serie de complicaciones potencialmente serias y no bien estudiadas.

Objetivo: describir la frecuencia reportada en la literatura de edema pulmonar en cirugía laparoscópica y en colecistectomía laparoscópica.

Metodología: estudio descriptivo de evidencia (revisión sistemática), utilizando las bases de datos Medline (PubMed, NLM), OVID y CINAHL. Dos revisores independientes evaluaron la pertinencia de los artículos, a partir de los resúmenes, y sobre ellos se hizo una revisión en texto completo del nivel de evidencia y la frecuencia de presentación de las complicaciones y específicamente del edema pulmonar. Se describe la mediana de frecuencia informada en la literatura (\%), de la presentación del edema pulmonar en cirugía laparoscópica.

Resultados: se encontraron 58 artículos (39 en Medline y 19 en OVID) En CINAHL se encontraron

\footnotetext{
* Profesor Asociado, Facultad de Medicina, Universidad Nacional de Colombia. Correo electrónico: jheslavas@unal.edu.co

** Profesor Titular, Facultad de Medicina, Universidad Nacional de Colombia

*** Especialista Cirugía General. Clínica Vascular Navarra
}

3 que ya se habían encontrado con las estrategias previas. De estos, 2 fueron estudios observacionales analíticos, 13 observacionales descriptivos diferentes a series de caso y reportes de caso y 25 fueron series de casos o reporte de un caso. No se encontraron en texto completo 6 artículos y 12 fueron revisiones no sistemáticas. La frecuencia de presentación de edema pulmonar en cirugía laparoscópica osciló entre 0 y 16,6\% Me=1,4\% (IC95\% 0,19\%-2,3\%). En muchos de los casos no hubo claridad sobre la causalidad del edema pulmonar y se asoció con el evento más inmediato, precedente.

Conclusión: la mayoría de la evidencia disponible es tipo III y IV. Se requieren de estudios de vigilancia posmercadeo (Fase IV) para este tipo de tecnologías. Mientras tanto es relevante dar a conocer a los pacientes sobre la presentación del edema pulmonar como una potencial complicación de la cirugía laparoscópica.

Palabras clave: edema pulmonar, revisión académica, colecistectomía laparoscópica.

\section{SUMMARY}

Laparoscopic surgery is a technique that has been popularized in an exponential manner and to which has been recognized many benefits too. However, it 
has been related with several and potentially serious complications.

Objective: to describe the frequency of pulmonary edema in laparoscopic surgery and in laparoscopic cholecystectomy in published studies.

Methodology: descriptive study of evidence (systematic review), using the Medline, OVID and CINAHL databases. Two independent reviewers evaluated the relevance of papers from their abstracts and after, the level of evidence and frequency of the event on the paper in full text. The median of frequency (\%) in published papers is showed.

Results: 58 articles (39 from Medline and 19 from OVID) were found, from these, three papers had been found previously in CINAHL. Two were analytic observational studies, 13 descriptive observational studies (not case series or case reports) and 25 were case series and case reports. 6 papers were not available in full text and 12 were non-systematic reviews. The frequency of pulmonary edema was found to be between $0 \%$ and $16.6 \%$ in published papers, Median= 1.4\% (CI95\% 0.19\% - 2.3\%). There was not a clear evidence of causation of pulmonary edema in the evaluated articles, and it was related with the nearest precedent event.

Conclusions: level of evidence of the articles was between III and IV. Post-marketing surveys are required (Phase IV studies) for this kind of technology. Whilst, pulmonary edema should be considered as a potential complication of laparoscopic surgery.

Key words: pulmonary edema, review, systematic, cholecystectomy, laparoscopic

\section{CASO CLÍNICO}

Paciente de 35 años, mujer, con diagnósticos de colelitiasis y colecistitis incipiente sometida a colecistectomía laparoscópica de urgencia. Antecedentes positivos: cesárea segmentarea hace dos años bajo anestesia general sin complicaciones. En valoración preanestésica se cataloga como Estado Físico ASA 2U. Al ingreso a salas se encontró tensión arterial (TA): 120/58 mmHg, frecuencia cardiaca (FC): $100 \mathrm{l} / \mathrm{min}$. Se monitoriza con visocardioscopio
(DII), oxímetría de pulso $\left(\mathrm{SaO}_{2}\right)$, capnógrafo de toma lateral $\left(\mathrm{ETCO}_{2}\right)$ y monitor digital de presión arterial no invasiva. Se realiza inducción con oxígeno (100\%), fentanyl, lidocaina, propofol y rocuronio. Se realiza intubación orotraqueal, diez minutos luego de la inducción recibe tramadol, dipirona y metoclopramida. El mantenimiento se hace con isoflurano. Durante el transoperatorio la paciente es colocada en posición de Trendelenburg invertido y se maneja con neumoperitoneo con $\mathrm{CO}_{2}$, presión regulada máxima de $15 \mathrm{mmHg}$, sin alteraciones de las variables monitorizadas en el transoperatorio. Duración de la cirugía: 2 horas. Se aplicaron 1.500 cC de Hartmann ${ }^{\circledR}$ en el intraoperatorio. Al final de la cirugía, cinco minutos luego de retirar el neumoperitoneo y coincidente con el movimiento de la paciente y la aplicación de la reversión farmacológica de la relajación muscular (atropina $1 \mathrm{mg}$ y neostigmine 2,5 mg IV, diluido) la paciente hace episodio de taquicardia supraventricular de $130 \mathrm{l} / \mathrm{min}$ (TA:140/70 mmHg; $\mathrm{SaO}_{2}$ : 95\%), durante 2 minutos, que progresa a bradiarritmia (FC:38, TA: 65/20 mmHg, $\mathrm{SaO}_{2}$ : 85\%) y bloqueo A/V completo. Se aplica $1 \mathrm{mg}$ de atropina. Dado que no hay respuesta se aplica un miligramo adicional. Se aumenta Presión Inspiratoria Máxima (PIM) a $30 \mathrm{~cm} \mathrm{H_{2 }}$ O. Hay salida de material rosáceo por tubo endotraqueal, se hace el diagnóstico de edema pulmonar y se inicia Presión Positiva al final de la Expiración (PEEP) de $10 \mathrm{~cm} \mathrm{H}_{2} \mathrm{O}$, sin mejoría significativa de signos vitales. Se inicia adrenalina en infusión a 0,05 $\mathrm{mcg} / \mathrm{kg} / \mathrm{min}$, dopamina $5 \mathrm{mg} /$ $\mathrm{kg} / \mathrm{min}$. TA:90/60, $\mathrm{SaO}_{2}: 70 \%$, se aumenta PEEP a $15 \mathrm{~cm} \mathrm{H} \mathrm{H}_{2} \mathrm{O}$, recuperándose la $\mathrm{SaO}_{2}$ (94\%). Se incrementa la dosis de adrenalina $(0,1 \mathrm{mcg} / \mathrm{kg} / \mathrm{min})$. Se coloca catéter venoso central por vena yugular interna izquierda (PVC $10 \mathrm{mmHg}$ ); diagnósticos probables: 1. Arritmia Intraoperatoria, 2. Edema Pulmonar Secundario, 3. Enfermedad coronaria silente (IAM?), 4. Embolismo $\mathrm{CO}_{2}$ ? y 5. Tromboembolismo pulmonar?. La paciente es trasladada a la unidad de cuidado intensivo en donde se continúa manejo con $\mathrm{FiO}_{2}$ de 0,9, PEEP: $15 \mathrm{~cm} \mathrm{H}_{2} 0$ e igual 
manejo inotrópico durante las 10 horas siguientes. Allí se descarta infarto agudo de miocardio y la presencia de trombos en miembros inferiores. Ecocardiografía mostró hipoquinesia global e insuficiencia mitral GII, fracción de eyección (FE): 40\%. En el posoperatorio la paciente hace fiebre que se considera de origen infeccioso del sitio quirúrgico. Se adiciona manejo antibiótico empírico, con buena respuesta; se hace extubación a las 60 horas del evento. Se da salida para la casa al quinto día posoperatorio.

Se plantea la pregunta: ¿Cuál es la frecuencia del edema pulmonar en cirugía endoscópica, específicamente en colecistectomía laparoscópica y qué factores están asociados a su aparición?

\section{METODOLOGÍA}

Con la anterior pregunta en mente se determinaron las siguientes palabras clave: laparoscopy, cholecystectomy, complications, pulmonary edema. Se hizo una búsqueda en las bases electrónicas de datos Medline (PubMed) , Ovid y CINAHL. En total se encontraron 58 artículos, 39 en Medline y 19 en Ovid.

Bajo el término de cirugía endoscópica se agrupó la laparoscopia y la histeroscopia, ya fueran diagnósticas u operatorias.

Dos revisores de manera independiente evaluaron la pertinencia de los artículos a partir de los resúmenes. Los artículos considerados pertinentes fueron posteriormente analizados en texto completo, para evaluar la validez y los resultados. La calificación de la evidencia se hizo siguiendo la siguiente clasificación sugerida por los autores: Tipo I: experimentos y meta-análisis de alta calidad; Tipo II: observacionales analíticos; Tipo III: observacionales descriptivos (diferentes a series de casos y reportes de caso) y cuasi-experimentales (no aleatorizados o pseudoaleatorizados); Tipo IV: series de casos y reportes de caso; Tipo V: revisiones de tema no sistemáticas.

\section{RESUILTADOS}

El edema pulmonar se ha descrito en 58 estudios de pacientes de cirugía endoscópica desde 1975 hasta la fecha, como complicación directa de la técnica o del riesgo inherente al estado del paciente, así como también al uso de ciertas técnicas y procedimientos en el manejo de los pacientes. ${ }^{1-39}$ Diez estudios fueron en el campo específico de la colecistectomía laparoscópica, , 10,11,14,20,21,26,27,30,34,35 de los cuales 9 describen la presencia de esta complicación en reportes de caso hasta grandes series de casos. (tabla 1) Los pacientes incluidos en las publicaciones son muy disímiles y la evidencia aportada, por ende, es muy diferente.

\section{Frecuencia del edema pulmonar en cirugía laparoscópica}

La frecuencia de edema pulmonar relacionada con cirugías laparoscópicas oscila entre 0\% y 16,6\%. La mayor frecuencia es reportada en una serie de casos en pacientes ancianas sometidas a enterolitotomía laparoscópica. ${ }^{33}$ Las series mas grandes reportan, sobre 17.298 pacientes sometidas a histeroscopia, una frecuencia entre el $0,34 \%$ y $0,14 \%{ }^{12}$ y, sobre 393 sometidas a laparoscopia de urgencia, una frecuencia de $0,5 \% .{ }^{19}$ En 35 pacientes sometidas a miomectomía por resectoscopio se encuentra una frecuencia del 5\%. ${ }^{28}$ La mediana de frecuencia de presentación del edema pulmonar en todos los artículos en los que se presentó más de un caso ( $\mathrm{n}=25$ artículos) fue de 1,4\% (IC95\% 0,19\%-2,3\%).

\section{Edema pulmonar y colecistectomía laparoscópica}

De los 10 artículos encontrados, 4 corresponden a reportes de caso (Nivel de evidencia IV) $)^{14,20,30,34}$, tres series de casos (Nivel de evidencia IV) ${ }^{21,27,35}$ y una revisión narrativa (Nivel de evidencia V) ${ }^{26}$ Uno de los artículos no se encontró ${ }^{11}$ y el otro es un estudio fisiopatológico. ${ }^{10}$ La frecuencia de presentación del edema pulmonar en las series de casos va desde $0 \%{ }^{27}$ hasta $4,47 \%$ en pacientes octogenarios. ${ }^{35} \mathrm{De}$ éstos, Shanta y cols. describen la presentación de una serie diversa de complicaciones relacionadas con la colecistectomía laparoscópica que incluyen el enfisema subcutáneo masivo, la bradicardia, el 
Tabla 1. Resumen de artículos en los que se presenta edema pulmonar bajo cirugía laparoscópica.

\begin{tabular}{|c|c|c|c|c|c|}
\hline Autor & $\begin{array}{l}\text { Evidencia } \\
\text { Tipo }\end{array}$ & Encontrado & $\mathbf{n}$ & $\begin{array}{l}\text { (n) \% en el } \\
\text { estudio }\end{array}$ & Relación \\
\hline \multicolumn{6}{|c|}{ Medline-PubMed } \\
\hline 1 & & No & & & \\
\hline 2 & & No & & & \\
\hline 3 & IV & Sí & 1 & (1) 100 & Embolismo $\mathrm{CO}_{2}$ \\
\hline 4 & IV & Sí & 284 & (2) 0,7 & Sobrecarga de líquidos \\
\hline 5 & IV & Sí & 1 & (1) 100 & Embolismo $\mathrm{CO}_{2}$ \\
\hline 6 & V (carta) & Sí & & & \\
\hline 7 & IV & Sí & 1 & (1) 100 & Dextrano intrauterino \\
\hline 8 & IV & Sí & 1 & (1) 100 & Tratamiento con $\mathrm{O}_{2}$ hiperbárico \\
\hline 9 & IV & Sí & 1 & (1) 100 & Cardiomiopatía \\
\hline 10 & IV & Sí & 27 & & No relacionado \\
\hline 11 & IV & No & & & \\
\hline 12 & III & Sí & 17.298 & (24) 0,141 & $\begin{array}{l}\text { Datos para 1991. En } 1988 \text { refieren } \\
0,34 \% \text { de edema pulmonar. Lo rela- } \\
\text { cionan con intoxicación hídrica. Hubo } \\
\text { tres casos de embolismo de } \mathrm{CO}_{2} \text { en } \\
1991(0,017 \%)\end{array}$ \\
\hline 13 & IV & No & & & \\
\hline 14 & IV & Sí & 1 & (1) 100 & Falla cardiaca \\
\hline 15 & IV & Sí & 1 & (1) 100 & Colapso cardiovascular \\
\hline 16 & IV & Sí & 1 & (1) 100 & Embolismo de $\mathrm{CO}_{2}$ \\
\hline 17 & IV & Sí & 1 & (1) 100 & $\begin{array}{l}\text { Reacción alérgica } \\
\text { al Dextrano } 70 \text { (32\%) }\end{array}$ \\
\hline 18 & III & Sí & 115 & (1) 0,86 & No se menciona \\
\hline 19 & III & Sí & 7.988 & (1) 0,012 & $\begin{array}{l}\text { Enfermedad miocárdica. } \\
\text { Un caso adicional } \\
\text { de falla respiratoria }\end{array}$ \\
\hline 20 & IV & Sí & 1 & 0 & Se revisa el tema. \\
\hline 21 & IV & Sí & 29 & (1) 3,44 & Pacientes mayores de 75 años \\
\hline 22 & IV & Sí & 1 & (1) 100 & Toracotomía previa \\
\hline 23 & IV & Sí & 1 & (1) 100 & $\begin{array}{l}\text { Inyección de azul de } \\
\text { metileno intrauterino }\end{array}$ \\
\hline 24 & IV & Sí & 1 & (1) 100 & $\begin{array}{l}\text { Inyección de vasopresina en pared } \\
\text { uterina. Presentó previamente } \\
\text { bradicardia y bloqueo A/V }\end{array}$ \\
\hline 25 & IV & Sí & 1 & (1) 100 & $\begin{array}{l}\text { Embolismo de aire durante } \\
\text { histeroscopía. }\end{array}$ \\
\hline 26 & $\mathrm{~V}$ & Sí & & & Revisión de tema \\
\hline 27 & III & Sí & 77 & (0) 0 & $\begin{array}{l}24 \text { hospitalizados, } 61 \text { ambulatorios, } \\
\text { mayores } 70 \text { años. No complicaciones }\end{array}$ \\
\hline 28 & II & Sí & 136 & (2) 1,47 & $\begin{array}{l}\text { En pacientes bajo miomectomía } \\
\text { resectoscópica. En los de laparoscópica } \\
\text { no hubo edema pulmonar. }\end{array}$ \\
\hline 29 & IV & Sí & 1 & (1) 100 & $\begin{array}{l}\text { Crisis adrenérgica } \\
\text { (feocromocitoma). La crisis ocurrió } \\
\text { durante el neumoperitoneo }\end{array}$ \\
\hline 30 & IV & Sí & 1 & (0) 0 & $\begin{array}{l}\text { Se revisa tema. Mediastinitis } \\
\text { por IOT difícil }\end{array}$ \\
\hline 31 & IV & No & 39 & & \\
\hline
\end{tabular}




\begin{tabular}{|c|c|c|c|c|c|}
\hline Autor & $\begin{array}{l}\text { Evidencia } \\
\text { Tipo }\end{array}$ & Encontrado & $\mathbf{n}$ & $\begin{array}{l}\text { (n) } \% \text { en el } \\
\text { estudio }\end{array}$ & Relación \\
\hline \multicolumn{6}{|c|}{ Medline-PubMed } \\
\hline 32 & III & Sí & 100 & (1) 100 & Desconocen la causa \\
\hline 33 & IV & Sí & 6 & (1) 16,6 & Paciente hace edema pulmonar y sepsis \\
\hline 34 & IV & Sí & 1 & (1) 100 & $\begin{array}{l}\text { Múltiples factores. Relacionan } \\
\text { hipertensión abdominal }\end{array}$ \\
\hline 35 & III & Sí & 67 & (3) 4,47 & Mayores de 80 años \\
\hline 36 & II & Sí & 47 & (1) 2,12 & $\begin{array}{l}\text { Manejo agresivo de líquidos. Todos } \\
\text { para resección de feocromocitoma }\end{array}$ \\
\hline 37 & IV & Sí & 1 & (1) 100 & Transfusión de plasma fresco congelado \\
\hline 38 & $\mathrm{~V}$ & No (carta) & & & \\
\hline 39 & II & Sí & 42 & (1) 2,3 & Falla renal crónica \\
\hline \multicolumn{6}{|c|}{ OVID } \\
\hline 50 & $\mathrm{~V}$ & Sí & & & Revisión \\
\hline 51 & $\mathrm{~V}$ & Sí & & & Revisión \\
\hline 52 & $\mathrm{~V}$ & Sí & & & Revisión \\
\hline 53 & $\mathrm{~V}$ & Sí & & & Revisión \\
\hline 54 & $\mathrm{~V}$ & Sí & & & Revisión \\
\hline 40 & $\mathrm{~V}$ & Sí & & & $\begin{array}{l}\text { Revisión, menciona experimento en } \\
\text { cerdos, } 2 \text { veces más edema pulmo- } \\
\text { nar cuando presión abdominal } \geq 20 \\
\text { mmHg. Sugiere fisiopatología }\end{array}$ \\
\hline 55 & $\mathrm{~V}$ & Sí & & & Revisión \\
\hline 48 & III & Sí & 129 & (1) 0,77 & Falla cardiaca, cáncer ginecológico \\
\hline 56 & III & Sí & 54 & (1) 1,85 & No se menciona \\
\hline 57 & I & Sí & 51 & (1) 1,96 & No se menciona. Referencia a Healzer \\
\hline 46 & III & Sí & 16 & (0) 0 & $\begin{array}{l}\text { Mencionan cambios hemodinámicos } \\
\text { de insuficiencia mitral, tricuspidea, } \\
\text { pulmonar y aórtica en sujetos sanos, } \\
\text { durante laparoscopia. }\end{array}$ \\
\hline 58 & III & Sí & 17 & (0) 0 & $\begin{array}{l}\text { Se analiza mecánica respiratoria } \\
\text { durante laparoscopia }\end{array}$ \\
\hline 41 & III & Sí & 63 & (2) 3,17 & Sobrecarga hídrica $(>13$ cc/kg/h) \\
\hline 59 & II & Sí & 50 & (2) 4 & $\begin{array}{l}\text { Sobrecarga hídrica. (grupo de } \\
\text { laparoscopia) }\end{array}$ \\
\hline 60 & IV & Sí & 2 & (0) 0 & $\begin{array}{l}\text { Explican signos de embolismo de } \mathrm{CO}_{2} \\
\text { como hipotensión, disrritmias, soplos, } \\
\text { cianosis, edema pulmonar y paro }\end{array}$ \\
\hline 61 & III & Sí & 84 & (2) 2,38 & No mencionan \\
\hline 62 & III & Sí & 30 & (0) 0 & $\begin{array}{l}\text { Relacionan cambios pulmonares } \\
\text { durante laparoscopia. }\end{array}$ \\
\hline 49 & IV & Sí & 8 & (1) 12,5 & $\begin{array}{l}77 \text { años, falla cardiaca, cáncer } \\
\text { de ovario }\end{array}$ \\
\hline 44 & IV & Sí & 10 & (1) 10 & Sobrecarga hídrica \\
\hline
\end{tabular}


malfuncionamiento de los oxímetros, el edema pulmonar, la intubación endobronquial y la caída del paciente, estos dos últimos relacionados con el manejo de las posiciones del paciente durante la cirugía (Nivel de evidencia IV). ${ }^{11}$

En uno de los reportes de caso relacionan la asociación de la presentación del edema pulmonar con el neumoperitoneo y la infusión de líquidos ${ }^{20} \mathrm{y}$ en otro, con falla cardiaca en una mujer de 59 años de edad, quien la desarrolla de manera súbita en la recuperación. En éste, luego de revisar las posibles causas como la extrema posición de Trendelenburg, el embolismo de $\mathrm{CO}_{2}$, los líquidos de irrigación peritoneal, la enfermedad cardiopulmonar, la obesidad, las reacciones adversas a medicamentos y edema de presión negativa, concluyen por exclusión, que la elevada presión intrabdominal que se usó en el caso descrito $(20 \mathrm{mmHg})$ podría estar relacionada y sugieren no usar presiones de neumoperitoneo superiores a $15 \mathrm{mmHg} .{ }^{34}$

Una de las series de casos con 29 colecistectomías realizadas en pacientes mayores de 75 años, de los cuales 10 requirieron drenaje pancreático adicional, tuvo una mortalidad global de 6,2\% $(n=2)$, uno de los pacientes que falleció, había desarrollado edema pulmonar agudo tres días después de la cirugía y posteriormente taponamiento cardiaco, que fue su causa de muerte; el otro, un paciente que desarrolló tromboembolismo cerebral en el sexto día posoperatorio (Nivel de evidencia IV). ${ }^{21}$ Otra de las series de casos (Tipo IV) ocurrió en 67 pacientes octogenarios, 38 de los cuales tenían alguna complicación preoperatoria (colecistitis, 15, pancreatitis biliar, 17, colangitis, 3 , ictericia obstructiva, 3 y que fueron ASA 3 o 4). El 18\% de los pacientes presentaron complicaciones entre las que se mencionan edema pulmonar en $3(4,4 \%)$, infarto del miocardio en 1 , atelectasias en 2 , lesión de la vía biliar en 1, infección del tracto urinario en 2 , infección de sitio quirúrgico en 2 y hematoma infectado intrabdominal en $1 .^{35}$

En la revisión narrativa (Tipo V) se describen una serie de complicaciones potencialmente serias relacionadas con la colecistectomía laparoscópica, dentro de las que incluyen: efectos cardiopulmonares que se asocian con la hipercapnia y la presión intrabdominal elevada, tendencia a acidosis relacionada con la absorción peritoneal de $\mathrm{CO}_{2}$, enfisema subcutáneo, neumotórax, neumomediastino y neumopericardio. Entre los efectos cardiacos mencionan además, las arritmias, la hipotensión, el paro cardiaco, el embolismo gaseoso, el edema pulmonar y la isquemia miocárdica o el infarto. Concluyen mencionando que aunque son complicaciones raras, son potencialmente graves y letales. ${ }^{26}$

En los artículos encontrados, dos de ellos revisan los cambios pulmonares durante la laparoscopia y los posibles mecanismos fisiopatológicos que llevan al edema pulmonar, entre los que se describen el aumento de las presiones de llenado pulmonar y la disminución del aclaramiento linfático pulmonar durante la fase de hipertensión abdominal como probables explicaciones..$^{10,40} \mathrm{En}$ un artículo hacen énfasis en la aplicación de volúmenes mayores de $13 \mathrm{cc} / \mathrm{kg} / \mathrm{h}$ como los responsables del edema pulmonar. ${ }^{41}$

\section{DISCUSIÓN}

Aunque al clasificar la evidencia se priorizan los artículos analíticos sobre los descriptivos, se debe destacar que el enfoque analítico de los artículos no está hecho sobre la presentación de complicaciones entre grupos de comparación. Las complicaciones no son el eje central de los estudios encontrados y por lo mismo, no se plantea en ellos la realización a priori de pruebas de hipótesis.

Si se considera la temporalidad como elemento sugerente de causalidad primordial y se ubica a la laparoscopia como factor de riesgo para el desarrollo de edema pulmonar, la evidencia de los artículos encontrados es de muy bajo nivel y no permite clarificarla. Adicionalmente, solo es posible considerar con la evidencia disponible la frecuencia de presentación de este evento, en series de casos.

En una revisión recientemente publicada sobre el riesgo relacionado con la anestesia, se recopila una serie de estimaciones sobre la probabilidad que ocurran 
eventos de morbilidad o mortalidad relacionados con anestesia, pero no incluye un ítem para describir el edema pulmonar como una complicación esperada del manejo anestésico. ${ }^{42}$ Entre los eventos etiológicos encontrados en esta revisión se relacionó el edema pulmonar con la sobrecarga hídrica. ${ }^{12,436}$ Este hecho también se ha mencionado por algunos autores, haciendo énfasis en la reabsorción de líquidos de la irrigación peritoneal como posibles causas de la sobrecarga. ${ }^{13}$ Otros factores que favorecerían el diagnóstico de sobrecarga hídrica, son: la compresión de la circulación en el hemiabdomen superior, el efecto de "torniquete venoso abdominal" que puede ejercer la hipertensión abdominal y la posición de Trendelenburg invertido que disminuye aún más el retorno venoso de miembros inferiores, que facilitarían la posibilidad de que, al liberar el "torniquete abdominal" se dé una restauración de la circulación venosa suprimida y que ocurra un efecto de infusión masiva de líquidos, la cual momentáneamente se comportaría como una sobrecarga hídrica relativa.

Es llamativo que solo tres artículos relacionen el caso con el embolismo de $\mathrm{CO}_{2}{ }^{3,5,16}$ probablemente porque no ha sido considerado habitualmente como agente causal y porque el diagnóstico se debe hacer por exclusión. El embolismo de $\mathrm{CO}_{2}$ es una entidad plausible y de la cual se ha reportado mortalidad en cirugías laparoscópicas ${ }^{3,43}$ y también a nivel experimental. ${ }^{44,45}$

Un estudio de observación con ecocardiografía, de los cambios hemodinámicos durante la laparoscopia reportó en sujetos sanos, la presencia de insuficiencia mitral aórtica, tricuspidea y pulmonar durante el evento, destacando la posibilidad de complicaciones serias en pacientes previamente enfermos y que se sometan a esta forma de intervención. ${ }^{46}$ Adicionalmente en una revisión del tema, se encontró que en un experimento de laboratorio con cerdos, los que se manejaban con presiones intraperitoneales de 20 $\mathrm{mmHg}$, desarrollaban 2 veces más edema pulmonar que los que tenían presiones menores. ${ }^{47}$ En un reporte de caso consideraron el aumento de la presión intrabdominal como la causa del edema pulmonar. ${ }^{14}$
Otros eventos temporalmente relacionados de manera directa por su inmediatez son la inyección de vasopresina, de azul de metileno o de dextrano. 17,23,24 Otros factores que han sido relacionados son los factores inherentes al paciente: cáncer, falla cardiaca, edad, miocardiopatía. ${ }^{9} 14,19,48,49$

Se deben estudiar más a fondo las repercusiones fisiológicas de los cambios hidrostáticos y pulmonares relacionados con esta cirugía, y desde el punto de vista epidemiológico, establecer estudios de fase IV, como se hace con los medicamentos, a fin de tener un registro más detallado de las potenciales complicaciones y riesgos que puede conllevar la cirugía laparoscópica y específicamente la colecistectomía.

\section{REFERENCIAS}

1. Girotti M, Schaer AE. Complications of gynecologic laparoscopy. Riv Ital Ginecol 1975;56:49-54.

2. Imran M, Yashari M, Slate WG. Laparoscopy and some of its hazards. Del Med J 1976;48:71-4, 76.

3. Root B, Levy MN, Pollack S, Lubert M, Pathak K. Gas embolism death after laparoscopy delayed by "trapping” in portal circulation. Anesth Analg 1978;57:232-7.

4. Helvacioglu A, Long EM Jr, Yang SL. Ectopic pregnancy. An eight-year review. J Reprod Med 1979;22:87-92.

5. Desai S, Roaf E, Liu P. Acute pulmonary edema during laparoscopy. Anesth Analg 1982;61:699-700.

6. Lamb JD. Aspiration or carbon dioxide embolism? Anesth Analg 1983;62:368.

7. Zbella EA, Moise J, Carson SA. Noncardiogenic pulmonary edema secondary to intrauterine instillation of 32\% dextran 70. Fertil Steril 1985;43:479-80.

8. McGrath BJ, Zimmerman JE, Williams JF, Parmet J. Carbon dioxide embolism treated with hyperbaric oxygen. Can J Anaesth 1989;36:586-9.

9. Hill GA, Perry SM, Herbert CM 3rd, Wentz AC. Cardiomyopathy in a patient undergoing laparoscopy for oocyte retrieval during in vitro fertilization/embryo transfer. A case report. J Reprod Med 1990;35:741-3.

10. McAllister JD, D'Altorio RA, Snyder A. CT findings after uncomplicated percutaneous laparoscopic cholecystectomy. J Comput Assist Tomogr 1991;15:770-2.

11. Shantha TR, Harden J. Laparoscopic cholecystectomy: anesthesia-related complications and guidelines. Surg Laparosc Endosc 1991;1:173-8. 
12. Hulka JF, Peterson HB, Phillips JM, Surrey MW. Operative hysteroscopy. American Association of Gynecologic Laparoscopists 1991 membership survey. J Reprod Med 1993;38:572-3.

13. Healzer JM, Nezhat C, Brodsky JB, Brock-Utne JG, Seidman DS. Pulmonary edema after absorbing crystalloid irrigating fluid during laparoscopy. Anesth Analg 1994;78:1207.

14. Spain K. Congestive heart failure associated with endoscopic cholecystectomy: a case report. AANA J 1994;62:46-8.

15. Wu MH, Lin CC, Yang YC, Chou CY. Cardiovascular collapse during gynecologic laparoscopy complicated by pulmonary edema: report of a case. J Formos Med Assoc 1994;93:629-32.

16. Gillart T, Bazin JE, Bonnard M, Schoeffler P. Pulmonary interstitial edema after probable carbon dioxide embolism during laparoscopy. Surg Laparosc Endosc 1995;5:327-9.

17. Huhn AM. Anaphylactic reactions to high molecular weight dextran during hysteroscopic surgery. CRNA 1995;6:167-71.

18. Khoury N. A comparative study of laparoscopic extraperitoneal and transabdominal preperitoneal herniorrhaphy. J Laparoendosc Surg 1995;5:349-55.

19. Martin-Vivaldi R, Nogueras F, Garcia Montero M, Quintero D, De Teresa FJ. [Emergency laparoscopy. A 20year experience]. Rev Esp Enferm Dig 1995;87:305-8.

20. Schmelzer C, Stone NL. Laparoscopic cholecystectomy in the cardiac patient: a case study. J Post Anesth Nurs 1995;10:18-20.

21. Ballesta Lopez C, Vila XB, Mato R, Gimenez A, Ruggiero R. [Laparoscopic surgery in patients over 75 years of age]. Minerva Chir 1996;51:1011-5.

22. Brown HS, Nezhat F, Nezhat C, Levy JS, Maffett M. Acute pulmonary edema complicating diagnostic laparoscopy preceded by thoracotomy. South Med J 1996;89:1217-9.

23. Trikha A, Mohan V, Kashyap L, Saxena A. Pulmonary edema following intrauterine methylene blue injection. Acta Anaesthesiol Scand 1996;40:382-4.

24. Tulandi T, Beique F, Kimia M. Pulmonary edema: a complication of local injection of vasopressin at laparoscopy. Fertil Steril 1996;66:478-80.

25. Behnia R, Holley HS, Milad M. Successful early intervention in air embolism during hysteroscopy. J Clin Anesth 1997;9:248-50.
26. Sharma KC, Kabinoff G, Ducheine Y, Tierney J, Brandstetter RD. Laparoscopic surgery and its potential for medical complications. Heart Lung 1997;26:52-64; quiz 65-7.

27. Voitk AJ. Is outpatient cholecystectomy safe for the higher-risk elective patient? Surg Endosc 1997;11:1147-9.

28. Park KH, Chung JE, Kim JY, Kim YT. Endoscopic management of uterine myoma. Yonsei Med J 1999;40:583-8.

29. Tauzin-Fin P, Hilbert G, Krol-Houdek M, Gosse $\mathrm{P}$, Maurette P. Mydriasis and acute pulmonary oedema complicating laparoscopic removal of phaechromocytoma. Anaesth Intensive Care 1999;27:646-9.

30. Hejjel L, Mecseky L, Szabo Z, Gal I. Fatal mediastinitis after routine laparoscopic cholecystectomy. Surg Endosc 2000;14:296.

31. Lertakyamanee N, Lertakyamanee J, Somprakit P, Nimmanwudipong T, Buranakitjaroen P, Bhavakula K, et al. Surgery and anesthesia for pheochromocytoma-a series of 40 operations. J Med Assoc Thai 2000;83:921-7.

32. Bernal-Gomez R, Olivares-Ontiveros O, GarciaVazquez A, Silva-Sanchez V, Noyola-Cedillo S, Quezada-Salcedo JE, et al. [Laparoscopic management of gastroesophageal reflux disease. Experience with 100 cases]. Rev Gastroenterol Mex 2001;66:80-5.

33. Ferraina P, Gancedo MC, Elli F, Nallar M, Ferraro A, Sarotto L, et al. Video-assisted laparoscopic enterolithotomy: new technique in the surgical management of gallstone ileus. Surg Laparosc Endosc Percutan Tech 2003;13:83-7.

34. Giaquinto D, Swigar K, Johnson MD. Acute congestive heart failure after laparoscopic cholecystectomy: a case report. AANA J 2003;71:17-22.

35. Hazzan D, Geron N, Golijanin D, Reissman P, Shiloni E. Laparoscopic cholecystectomy in octogenarians. Surg Endosc 2003;17:773-6.

36. Jaroszewski DE, Tessier DJ, Schlinkert RT, Grant CS, Thompson GB, van Heerden JA, et al. Laparoscopic adrenalectomy for pheochromocytoma. Mayo Clin Proc 2003;78:1501-4.

37. Barrio J, Carrera MD, Sanmiguel G, Garcia V. [Acute lung injury related to transfusion of fresh frozen plasma]. Rev Esp Anestesiol Reanim 2004;51:342-5. 
38. Davies S, Mordani K. Anesthetic management of laparoscopic surgery for twin to twin transfusion syndrome. Can J Anaesth 2004;51:945-6.

39. Yagisawa T, Ito F, Ishikawa N, Matsuda K, Onitsuka S, Goya N, et al. Retroperitoneoscopic adrenalectomy: lateral versus posterior approach. J Endourol 2004;18:661-4.

40. Malbrain ML, Deeren D, De Potter TJ. Intraabdominal hypertension in the critically ill: it is time to pay attention. Curr Opin Crit Care 2005;11:156-71.

41. Wolf JSJ, Monk TG, McDougall EM, McClennan BL, Clayman RV. The extraperitoneal approach and subcutaneous emphysema are associated with greater absorption of carbon dioxide duringlaparoscopic renal surgery. J Urol 1995;154:959-63.

42. Jenkins K, Baker AB. Consent and anaesthetic risk. Anaesthesia 2003;58:962-84.

43. Hanney RM, Alle KM, Cregan PC. Major vascular injury and laparoscopy. Aust N Z J Surg 1995;65:533-5.

44. Dion YM, Gracia CR, Estakhri M, Demalsy JC, Douville Y, Piccinini E, et al. Totally laparoscopic aortobifemoral bypass: a review of 10 patients. Surg Laparosc Endosc 1998;8:165-70.

45. Yau P, Watson DI, Lafullarde T, Jamieson GG. Experimental study of effect of embolism of different laparoscopy insufflation gases. J Laparoendosc Adv Surg Tech A 2000;10:211-6.

46. Fahy BG, Hasnain JU, Flowers JL, Plotkin JS, Odonkor P, Ferguson MK. Transesophageal echocardiographic detection of gas embolism and cardiac valvular dysfunction during laparoscopic nephrectomy. Anesth Analg 1999;88:500-4.

47. Quintel M, Pelosi P, Caironi P, Meinhardt JP, Luecke T, Herrmann $\mathrm{P}$, et al. An increase of abdominal pressure increases pulmonary edema in oleic acid-induced lung injury. Am J Respir Crit Care Med 2004;169:534-41.

48. Chi DS, Abu-Rustum NR, Sonoda Y, Awtrey C, Hummer A, Venkatraman ES, et al. Ten-year experience with laparoscopy on a gynecologic oncology service: analysis of risk factors for complications and conversion to laparotomy. Am J Obstet Gynecol 2004;191:1138-45.

49. Amara DP, Nezhat C, Teng NN, Nezhat F, Rosati M. Operative laparoscopy in the management of ovarian cancer. Surg Laparosc Endosc 1996;6:38-45.
50. Troy AM, Cunningham AJ. Ambulatory surgery: an overview. Curr Opin Anaesthesiol 2002;15:647-57.

51. Ogunnaike BO, Jones SB, Jones DB, Provost D, Whitten CW. Anesthetic considerations for bariatric surgery. Anesth Analg 2002;95:1793-805.

52. Bradley LD. Complications in hysteroscopy: prevention, treatment and legal risk. Curr Opin Obstet Gynecol 2002;14:409-15

53. Coskun F, Salman MA. Anesthesia for operative endoscopy. Curr Opin Obstet Gynecol 2001;13:371-6.

54. Milad MP, Sankpal RS. Laparoscopic approaches to uterine leiomyomas. Clin Obstet Gynecol 2001;44:401-11

55. Magrina JF. Laparoscopic surgery for gynecologic cancers. Clin Obstet Gynecol 2000;43:619-40.

56. Chan DY, Cadeddu JA, Jarrett TW, Marshall FF, Kavoussi LR. Laparoscopic radical nephrectomy: cancer control for renal cell carcinoma. J Urol 2001;166:2095-9; discussion 2099-100.

57. Coloma M, Zhou T, White PF, Markowitz SD, Forestner JE. Fast-tracking after outpatient laparoscopy: reasons for failure after propofol, sevoflurane, and desflurane anesthesia. Anesth Analg 2001;93:112-5.

58. Fahy BG, Barnas GM, Nagle SE, Flowers JL, Njoku MJ, Agarwal M. Changes in lung and chest wall properties with abdominal insufflation of carbon dioxide are immediately reversible. Anesth Analg 1996;82:501-5.

59. Pessaux P, Regenet N, Tuech JJ, Rouge C, Bergamaschi R, Arnaud JP. Laparoscopic versus open cholecystectomy: a prospective comparative study in the elderly with acute cholecystitis. Surg Laparosc Endosc Percutan Tech 2001;11:252-5.

60. Noga J, Fredman B, Olsfanger D, Jedeikin R. Role of the anesthesiologist in the early diagnosis of lifethreatening complications during laparoscopic surgery. Surg Laparosc Endosc 1997;7:63-5.

61. Rhodes M, Rudd M, Nathanson L, Fielding G, Siu S, Hewett P, et al. Laparoscopic anterior resection: a consecutive series of 84 patients. Surg Laparosc Endosc 1996;6:213-7.

62. Rishimani AS, Gautam SC. Hemodynamic and respiratory changes during laparoscopic cholecystectomy with high and reduced intraabdominal pressure. Surg Laparosc Endosc 1996;6(3):201-4. 